\title{
La Resistenza in Il Clandestino di Mario Tobino
}

\author{
Ahmed Mohamed Al Qarni \\ Assistente nel dipartimento italiano
}

Nell'introduzione presenteremo un quadro generale delle condizioni storiche e sociali dell'Italia del primo e secondo Novecento. Allargheremo poi la prospettiva al clima culturale, prendendo in considerazione le caratteristiche speciali che hanno contrassegnato la produzione artistica nel periodo della Seconda Guerra mondiale per gettare più luce sulla trattazione specifica di Tobino e quella di altri scrittori a lui contemporanei, il che ci permetterà di controllare la posizione dello scrittore nel quadro della letteratura italiana del dopo guerra.

Procederemo poi a parlare degli importanti fattori storici, politici e sociali che hanno contribuito a maturare la personalità di Tobino e che hanno avuto un grande effetto sul suo carattere come scrittore: la libertà a lui offerta da parte del padre (anticonformista), la partecipazione alla guerra in Libia, l'ammirazione per Dante, Macchiavelli, Tacito, Manzoni ed altri, la collaborazione a periodici come "il Selvaggio", "l'Italiano" e "Letteratura", oltre all'aderenza all'Organizzazione dei giovani antifascisti a Viareggio come abbiamo già detto in precedenza.

Tale studio dei fattori sopraelencati, ci farà come guida per poter peneterarci nel mondo e nella profondità dell'animo del nostro autore, il che ci aiuterà a capire bene i vari elementi della Resistenza italiana contro la dittatura del tempo, inclusi nelle pagine dell'opera letteraria oggetto di questa tesi.

Nel Primo capitolo intitolato Resistenza cercheremo di esporre il tema più importante e spinoso della storia d'Italia del dopo guerra: la Resistenza. Tobino, attraverso le pagine de il Clandestino, ha sigillato attentamente la vera storia della Resistenza italiana contro la dittatura fascista. L'autore, nonostante la semplice trama, ambientata in uno spazio limitato (Medusa), e registrata in un breve arco di tempo, si è riuscito a presentare al 
mondo la meravigliosa storia eroica di un gruppo di ragazzi nella propria lotta per la liberazione nazionale:

«Durante gli anni immediatamente successivi alla Liberazione, nel clima promosso dalla politica culturale del Partito comunista ispirato alla collaborazione delle forze progressiste dell'antifascismo, sui quotidiani di sinistra, vengono pubblicati (e in tale modo largamente diffusi) numerosi racconti sulla resistenza partigiana $»^{\mathrm{i}}$.

\section{Fascismo e antifascismo}

In primo luogo, esamineremo il motore principale della Resistenza, cioè il fascismo. Questo, difatti, è il vero e l'unico avversario dei giovani clandestini. Il regime dittatoriale nutre o piuttosto anima con tanto odio e disprezzo lo spirito dei giovani, stimolandoli così a manifestarsi alla vendetta per il paese ma anche per loro stessi:

"I fascisti erano ancora intontiti, si andavano a trovare da casa a casa, forse facevano qualche proposito ma per il momento erano chiacchiere. ${ }^{\mathrm{ii}}$.

L'antifascismo, quindi, è il fenomeno della lotta armata che occupa una posizione considerevole nel quadro della storia italiana nel periodo seguente alla seconda guerra mondiale.

Antifascisti sono quegli uomini, di vasta esperienza politica e morale, che repudiano qualsiasi forma di esclusione o marginazione eseguita nei loro confronti da parte del regime fascista. Lottano essenzialmente non per ottenere fama, anzi perchè fedeli alla patria, fedeli al bene e alla giustizia, perciò sono disposti ad ogni sacrificio:

"Il risveglio improvviso contro l'oppressore di un popolo giustiziare dopo venti anni muti fu poi anche più facilmente interpretato dalla corrente moderata come operazione militare di tutto un popolo unito contro lo straniero in una sorta di palingenesi morale non politicamente e socialmente finalizzataii".

\section{Disperazione e speranza}

Un tema molto eminente nella narrativa tobiniana di argomento resistenziale è la Disperazione. 
Questa condizione psicologica trova un'atmosfera molto adatta tra i popoli oppressi o ben sottomessi da regimi dittatoriali: "Contro gli avversari politici il fascismo fu spietato, e socialisti, comunisti, militanti di «Giustizia e libertà », operai o intellettuali che fossero, conobbero a migliaia le carceri fasciste; e i più pericolosi, o quelli ritenuti più pericolosi, pagarono con la vita la loro opposizione..." ${ }^{\text {iv }}$.

In questo clima di restrizione dittatoriale del regime nasce la disperazione di Tobino e di tutti i suoi compagni antifascisti. Una sensazione che affligge tutta la vita dei personaggi di $\boldsymbol{I l}$ Clandestino fino all'inizio della lotta per la liberazione.

L'aderenza dei ragazzi all'Oraganizzazione della liberazione nazionale e le relazioni d'amicizia ad essa conseguente, suscitano nei giovani partigiani una lieve sensazione di speranza e di rigenerazione, che si esercita, però in un tempo di paure e di uccisioni: "Mentre la speranza canta nei cuori dei giovani personaggi del romanzo, senti dietro quel canto, dentro quel canto che è soprattutto di amore, le vestigia, i segni profondi della paura, della morte."v.

\section{La morte}

La presenza della morte in Il Clandestino e altre opere di Tobino, fa come un elemento essenziale nella sua realtà complessa. Questa realtà fatta di speranza e di disperazione, di morte e di vita; realtà mai accettata con un bell' umore, ma come un incubo sempre turbante. Questo elemento, infatti, diventa un'idea ossessionante per Tobino, motivo cardine della sua opera, ma non solo questo romanzo. Ne Il Clandestino, Tobino non racconta solo le vicende reali ed eroiche dei ragazzi viareggini, ma anche la loro giovinezza perduta e la loro morte immatura: "Il primo a morire fu Anselmo ${ }^{\text {vi }}$.

Studiando accuratamente l'opera tobiniana, appare esplicito che la morte ha lasciato la sua traccia costante sulla memoria dell'autore, il che lo ha massimamente portato a dare all'ultimo capitolo dell'opera un titolo con una diretta referenza al tema «Anche Anselmo se ne va».

\section{Secondo capitolo: La tecnica narrativa}


Nel secondo capitolo ci occuperemo dello studio dei mezzi tecnici di cui Tobino fa uso per esprimere le sue esperienze da clandestino ed i suoi pensieri.

In primo luogo, sottoporremo allo studio l'Autobiografismo: tecnica di base del romanzo, preso in esame e che fa come una cornice generale della narrazione.

Anche se Tobino non avesse, una volta, dichiarato: «Il periodo più bello della mia vita fu nel clandestino, nella lotta di liberazione nazionale, dove finalmente avevo la mia bandiera», lo si sarebbe ben capito dalla lettura de Il Clandestino, per il persuaso amore, l'entusiasmo, l'intima adesione che anima quelle pagine e ne costituisce la ragione prima ${ }^{\text {vii }}$.

L'esordio autobiografico, basato su una memoria fresca e ricca di esperienze e testimonianze dirette, ha fatto della narrazione un corpo cronachistico del tutto armonico, e non è affato ignorato l'impegno del lato narrativo:

Il tessuto cronachistico prevalente nell'esordio ricorrerà poi come una sorta di connettivo a fondere tra di loro le parti più propriamente narrative. Già in questa struttura, vista internamente, si avverte l'insieme della soluzione stilistica, di un interesse tutto particolare.. ${ }^{\text {viii. }}$.

Cercheremo anche di dimostrare che la memoria è il punto chiave della struttura autobiografica de Il Clandestino. La narrazione si fonda in maggior parte su una memoria fresca, già affollata e consolidata di testimonianze e di esperienze personali dell'autore.

Vale la pena menzionare che, nella storia della letteratura italiana, la narrativa ispirata alla Resistenza $o$ in genere all'antifascismo si considera come una letteratura dell'oggetto, cioè, viene composta dall'analisi di fatti e di personaggi del tutto reali: ecco perchè Il Clandestino di Tobino ha una struttura semplice e cronachistica, di narrazione classica con un tono piano: "C'è una piazza a Medusa che da tutti vien chiamata Piazza Grande, anche se possiede un altro nome" $e^{\text {"ix. }}$

Questo elemento autobiografico su cui è basato la narrazione del romanzo suscita un dibattito aperto fra i critici che lo accusano di essere solo un diario o un libro semplice a Medusa senza problemi drammatici, senza angosce attiranti; a tale proposito ci 
basterà citare solo queste parole di Adriano Seroni in difesa all'opera:

Mi è capitato più di una volta di sentir dire che Il Clandestino è sì un bel libro, ma che è un libro troppo semplice, semplicemente impostato e risolto, senza problemi, senza angosce, quasi senza dramma. No assolutamente: Il Clandestino non è un libro semplice, non scansa nè ignora i grossi problemi del nostro tempo, non è nè idillio nè bozzetto. ${ }^{x}$.

Il secondo punto si concentrerà sull'analisi letteraria dei personaggi.

E normale che in un romanzo autobiografico risulta molto frequente l'affollarsi dei personaggi su cui circolano essenzialmente le azioni. Tobino raccoglie ne Il Clandestino quasi tutti i tipi di personaggi presenti in Italia al tempo della Resistenza: ci sono i Comunisti, il Monarchico e l'aristocratico.

Cercheremo di fare un analisi totale di tali personaggi prendendo in considerazione i pensieri, le caratteristiche, il grado di influenza sullo scorrere delle azioni ecc...

Tobino riesce con tanta intelligenza espressiva a presentare dei personaggi particolari, reali nelle loro azioni e stati d'animo: Le carte rimaste conservano i nomi dei veri protagonisti. Tobino vuole raccontare davvero quella storia e non nasconde le loro identità: ${ }^{x i}$.

La figura di Tobino non è rappresentata esplicitamente sulle pagine del romanzo, anzi egli espone con tanta semplicità i suoi ideali ed i suoi commenti sui fatti sia in terza persona che dietro al personaggio del dottor Anselmo, il più opportuno alla posizione di Tobino riguardante alla Resistenza: posizione di razionalità e di sentimentalità:

Il personaggio di Anselmo, il più obbiettivamente vicino all'autore (in certi particolari quasi vezzeggiato), si delinea con indugiante felicità: non «politico», è già tutto nella gentile sequenza che lo rappresenta nei contatti con la pettinatrice Iole e le apprendiste di questa; nell'acquistare improvviso, alle parole della ragazza, la coscienza dei tempi. Le convinzioni politiche di Anselmo restano comunque fondate sul «sentimento», sulle «appassionate ragioni» ${ }^{\mathrm{xii}}$.

L'ultimo punto di questo capitolo sarà dedicato allo studio del linguaggio e dello stile. Cercheremo di evidenziare, sotto questo punto, i numerosi segni stilistici e linguistici che formano la scorrente stesura de Il Clandestino: ne individueremo alcuni 
esempi come la mancanza dell'uso delle inversioni soggetto predicato; gli inizi e le riprese nei diversi capitoli che collegano in maniera essenziale i fatti dell'elemento tempo: «C'è una piazza a Medusa ..», «In una serena notte d'estate 1943...».

A proposito dell'uso dell'onomastica e la toponomastica, la critica Paola Italia dice:

Lasciando intendere la presenza di una trama sotterranea resa ben visibile dai frequenti richiami tra i personaggi, dalla tenuta narrativa dell'insieme e da un effetto complessivo di incremento della suspense nei lettori, che vengono via via catturati e avvinti dalle vicende. ${ }^{\text {xiii }}$

Poi esamineremo il modo di narrazione di Tobino che evita le clausole ritmiche, le spezzature d'affetto. Non manca però qualche inserimento poetico che non ostacola per niente la successione dei fatti.

In effetti, i titoli dei capitoli sono tessuti in un modo logico ed armonico: leggendoli successivamente, un lettore certamente ne otterrà un'idea assai chiara di questo procedimento.

Nella Conclusione, presenteremo i risultati raggiunti dalla nostra ricerca sul tema della Resistenza che si considera assai importante nella storia della letteratura italiana. Cercheremo di esporre tutti i dati e le notizie raccolti rispetto ai personaggi clandestini: protagonisti del periodo storico e dell'opera di Tobino. 


\section{Bibliografia}

Rosario Contarino e Marcella Tedeschi, Dal fascismo alla Resistenza, Laterza, Città di Cartello, 1980. p. 200.

ii Mario Tobino, Il Clandestino, Arnoldo Mondadori Editore, Milano, 1962, p. 74

iii Rosario Contarino e Marcella Tedeschi, op. cit. p. 168

iv Giuseppe Petronio, L'attività letteraria in Italia, Palumbo, Firenze, 1980, p. 858.

v Gianni Grana, Novecento- I contemporanei- Gli scrittori e la cultura letteraria nella società italiana, Marzorati editore, Milano, 1979, p. 6648.

vi Ivi., p. 559.

${ }^{v i i}$ Felice Del Beccaro, Mario Tobino. Il Castoro, La Nuova Italia, Firenze, 1967, p. 103. viii Ivi., p. 107.

ix Mario Tobino, Opere scelte, "a cura di" Paola Italia, I Meridiani, Arnoldo Mondadori Editore, Milano, 2011, p. 755.

${ }^{x}$ Gianni Grana, op. cit., pp. 6651- 6652.

${ }^{x i}$ Paola Italia, Tobino_Clandestino-Libre. pdf., 2013, p.10, in WWW.Accademia.eu

xii Felice Del Beccaro, op. cit., p. 109.

xiii Paola Italia, op. cit., p. 12. 